\title{
COMUNICACIÓN AUDIOVISUAL Y LA NECESIDAD DE AMPLIAR SU DIFUSIÓN.
}

Jesús García Jiménez: Universidad Complutense de Madrid (España)

"Ojalá el cielo nos conceda la serenidad para aceptar las cosas que no se pueden cambiar, el coraje para cambiar todo lo que se puede y se debe, y la inteligencia para discernir entre ambas cosas" (Nieburg)

\section{Resumen}

La Comunicación audiovisual tiene una importancia creciente en nuestra sociedad. Cada pocos años nuevas formas, nuevas plataformas y nuevas ideas surgen para que la estimulación electrónica y video-sonora se inserte un poco más en la vida cotidiana del individuo, lo que sin duda lleva al auge de la selección individualizada de contenidos. Sin embargo esta importancia social no va acompañada de un aumento de su presencia, de su importancia como tal disciplina en el ámbito académico preuniversitario. Un periodo esencial para preparar a las generaciones venideras ante el bombardeo de estímulos al que van a ser sometidos durante toda su vida, de forma que puedan entender mejor el lenguaje empleado en estos medios y la intención con la que se utiliza. Ello no es sino consecuencia de una serie de males endémicos tanto de la producción científica como de la política educativa y los elementos relacionados intrínsecamente con la forma en que las instituciones universitarias -depositarias del conocimiento y los medios humanos (que no económicos) para remediar la situación- han sido administradas en las últimas décadas. La investigación en comunicación audiovisual debe racionalizarse para no perder esfuerzos en un bosque inacabable de iniciativas brillantes pero de escaso recorrido y aún menor efecto global.

Palabras clave: Comunicación Audiovisual, Universidad, reforma, estatuto científico, comunicólogo

\section{Extracontexto y contrasentido}

A finales del mes de Junio del 1999 se inauguraba en Budapest la Conferencia Mundial sobre la Ciencia, convocada por la UNESCO. Su lema: Ciencia para el siglo 
$X X I$, un nuevo compromiso. Hacía 20 años que no se convocaba una Conferencia de este tipo. Entre los 2.000 asistentes había científicos, gestores de política científica, representantes de más de 150 países y de 170 organizaciones no gubernamentales. La intención de la UNESCO y del Consejo Internacional de las Uniones Científicas, que actuó de organismo coordinador, era que al concluir los trabajos de la Conferencia el 1 de julio, se adoptase una Declaración Mundial sobre la Ciencia y el Uso del Saber Científico, así como un Marco General de Acción.Se trataba de potenciar el acceso de la población a los conocimientos científicos y de promover una alianza entre la ciencia y la sociedad, capaz de resolver los problemas del mundo de mañana.Se da la chocante paradoja de que la comunicación audiovisual, que caracteriza a la emergente cultura global de fin del milenio, es invocada en todos los foros como herramienta decisiva en la "sociedad del conocimiento" (y por supuesto, del conocimiento que hoy estudia la sociología de la ciencia) y, sin embargo, su intervención en el escenario de la ciencia se limita a servir a ésta de vehículo, pero no de objeto. La Conferencia propuso la creación en Londres de un Centro Internacional para la Comunicación Científica, que puede servir de nodo en el esfuerzo mundial por mejorar la comunicación científica, centrándose específicamente en el periodismo científico y en la popularización de la ciencia, pero es chocante, digo, que, cuando se trata de comunicación todo el mundo, incluso la ADC (Asociación para el Desarrollo de la Ciencia) parece olvidar que también su desarrollo depende de la investigación. Todo un contrasentido.

Se me ocurre que la razón de esta sinrazón estriba en un desajuste manifiesto entre la investigación científica (sobre todo la escasa investigación básica) y la investigación tecnológica. La investigación científica en materia de comunicación (y especialmente de comunicación audiovisual) se halla perdida en un bosque de iniciativas apresuradas y brillantísimas, promovidas por la industria, que van mermando el terreno del desarrollo científico e imponiendo a la sociedad rutinas de uso y consumo que no han sido suficientemente validadas. Hay que decir con toda tristeza que la Universidad, al menos la Universidad española, ha perdido a favor de la industria toda capacidad de iniciativa y de influencia.

\section{Preguntas radicales}


Ante este panorama uno siente la tentación de formularse algunas preguntas radicales: ¿Existe realmente una ciencia de la Comunicación Audiovisual?... ¿Es reconocida y cotizada por alguien?... ¿Sirve realmente para algo?...

La respuesta a preguntas de este tenor debe pasar por un análisis previo de las condiciones objetivas que relacionan a la Comunicación Audiovisual con los entes dedicados a la investigación y muy especialmente, es obvio, a la Universidad.

\section{Algunos males endémicos de la producción científica}

La definición de la Universidad como productora de ciencia parece hoy aquejada de algunos males, que están convirtiéndose en endémicos y afectan, no sólo a la política científica española, sino también a la orientación de la política científica de la Unión Europea. Entre esos males debemos citar el sometimiento de la investigación científica al desarrollo tecnológico; la neta orientación de la Universidad al mercado laboral; la consideración seudodemocrática de que la oferta universitaria ha de ser "pan para todos"; Y la profesionalización, entendida como banalización o suplantación de conocimientos, falsamente tenidos por científicos. Estos males endémicos son especialmente peligrosos y virulentos porque se revisten en las sociedades avanzadas de una cierta legitimación ideológica, como si una "Universidad para los mejores" fuera santo y seña de dictaduras oprobiosas que más nos vale olvidar para siempre.

\section{La Universidad del "pan para todos"}

España es un país que ha entrado en la modernidad, compartiendo estos tics desde el primer momento. Aquella política científica, precaria, improvisada e incluso tercermundista que practicaba hace apenas veinte o veinticinco años, ha sabido concentrar el esfuerzo en esta corriente y la industria española compite hoy con toda dignidad en patentes y en desarrollo tecnológico con los países más avanzados.

La universidad española, estimulada por la Ley, se ha abierto a las empresas a través de fundaciones mixtas y se ha abierto al mercado laboral, instigada por los fondos de la Unión Europea, a través de sus ofertas de títulos propios, que están amenazando con convertirse en una especie de universidad paralela. Efectivamente, 
la Universidad del "pan para todos", ya no basta para abrirse paso en el mercado laboral y, cuando una muchacha o un muchacho, que ha concluido su licenciatura, busca su primer empleo, lo sufre en sus propias carnes. Ya no tiene bastante con aducir su condición de titulado superior, sino que ha de mostrar otros avales, como los másters, algunos másteres, que en opinión de los empleadores y de la sociedad en general, legitiman más y mejor su preparación. Por si fuera poco, el descrédito generalizado de este modelo de universidad lleva a los empleadores a incluir en el baremo de selección preguntas estúpidas sobre la experiencia del candidato, como si este deber que sólo enseñan la edad, el trabajo y la vida fueran exigibles a un joven, que, al salir de las aulas, se asoma a la vida real.

\section{La reforma pendiente del sistema educativo}

Tradicionalmente construido a base de retazos e improvisaciones, el educativo no ha pasado de ser un mal sistema de instrucción y de enseñanza, incongruente y peligrosamente disociado de la ciencia, la tecnología, la comunicación y la cultura viva de actualidad. Desde la Ley de Instrucción Pública de Claudio Moyano, alcalde constitucional de Valladolid que se sublevó contra Espartero y propuso la Constitución de 1845 como forma de gobierno, este país lleva ciento cincuenta años reclamando para su sistema educativo un ejercicio de unidad, racionalidad, coherencia, calidad y modernidad. Al acabar el milenio, treinta años nos separan de la Ley General de Educación (1970) que ha sido por ahora el único intento serio, pero fallido. Lástima que el régimen franquista tuviera más interés en su propaganda demagógica que en su financiación y aplicación. Esta utilización demagógica de las decisiones en materia de educación es un mal hábito político que siguen cultivando los actuales responsables políticos en la España democrática.

\section{La política audiovisual española}

La política audiovisual española, no podía ser de otro modo, es un verdadero campo de agramante. En la nómina de los males innumerables que la aquejan, ya nos hemos habituado a incluir las críticas sobre las contradictorias e improvisadas medidas de promoción, sobre la política de subvenciones o sobre el sometimiento de la comunicación audiovisual a las veleidades del poder, al patrocinio comercial o a las leyes de la competencia desleal y salvaje. El criterio para medir el interés de los 
políticos y gobernantes por los grandes medios de la comunicación audiovisual parece ser, en efecto, la generosidad con que meten mano en el erario público para subvenir las necesidades de industrias subdesarrolladas y mal gestionadas. En una democracia avanzada como la nuestra cuadra mal la creencia desgraciadamente generalizada de que el desarrollo del universo audiovisual depende más de la subvención desleal e indiscriminada que de su inclusión preferente en los objetivos de una política que aspira al desarrollo científico y tecnológico, al reparto justo y equitativo de los bienes culturales y morales y al respeto leal a la iniciativa privada y a sus oportunidades de negocio. La redefinición de la política audiovisual exige racionalidad y rigor, equidad y competencia leal, esclarecimiento y coraje.

\section{El sector industrial de lo audiovisual}

El sector audiovisual es en su dimensión industrial precario, paradójico y dominado por tres grandes contradicciones. Limitado en sus dimensiones, artesanal en sus conceptos y azaroso en sus entornos de gestión y negocio, se inscribe al mismo tiempo inevitablemente en el contexto virtual y global de esos colosales conglomerados industriales, dominados por los moguls. Aparece el audiovisual como un sector que aspira a preservar el cofre sagrado de las identidades culturales y, a cambio de tan alta misión, reclama las dispensas arancelarias de una industria cultural protegida, que en muchas ocasiones estaría por demostrar.

Con ocasión del Acuerdo General sobre Aranceles Aduaneros y de Comercio (GATT) los profesionales reclamaron cláusulas de excepcionalidad para el audiovisual, más en concreto para el sector cinematográfico. El audiovisual es un sector considerado hoy por la Unión Europea como "estratégico", por ser patrimonio cultural del Viejo Continente y por el volumen económico que moviliza. Al finalizar el siglo este volumen está alcanzando los 35.000 millones de euros (más de 4'5 billones de pesetas actuales). Según los estudios de Bridge Media, la tasa de crecimiento del cine en Europa es superior a la de Estados Unidos y la industria cinematográfica española en los últimos años del milenio va a crecer en tomo al 20 por 100, más de lo previsto para el cine británico, por ejemplo. En el cine español se advierte, es un consuelo, un nuevo impulso de entusiasmo y creatividad de la mano de autores muy jóvenes. 


\section{Contradicciones}

Las contradicciones que aquejan al sector audiovisual llevan a sus actores a contemplar la magnitud global de su mercado industrial, a establecer como criterio productivo y competitivo la defensa de las identidades particulares y a denunciar al mismo tiempo su fragmentación en mercados nacionales y en industrias entecas y lampantes. ¿Cómo puede entenderse esto?... Los participantes en el Grupo Temático de la Conferencia Europea del Sector Audiovisual (2 julio, 1994) pedían reforzar urgentemente la industria cinematográfica, aquejada de fragmentación en mercados nacionales, empresas de dimensiones insuficientes, cuyos productos son de escasa circulación internacional, encerradas en una "espiral de déficit crónico" e incapaces de atraer capital europeo.

\section{Ciencia y creatividad}

En el rigor que debería presidir una regulación del sector audiovisual europeo también la propiedad intelectual plantea algunos problemas. La propiedad intelectual del autor es entendida como derecho patrimonial indivisible, en contra de los todopoderosos magnates de la industria de la comunicación y del espectáculo y de los poderosos grupos multimedia. En esta guerra desigual por el control del consumo audiovisual la SGAE practica un corporativismo a la defensiva, patente en el spot que hace un par de años difundía la televisión. Autores consagrados, como Martín Scorsese, sostenían el mismo punto de vista en las jornadas sobre Realidad Audiovisual Europea (Madrid, 1995). La creatividad es una planta que surge y crece espontáneamente en los dominios de lo audiovisual, como si, lejos de la investigación científica y de la práctica pedagógica, su verdadero y único suelo nutricio fuera el terreno privado de la intuición o de la ocurrencia. Y la Universidad, viéndolas venir.

\section{La identidad cultural como coartada}

Los programas EUREKA (para la promoción integral del espacio audiovisual europeo) y MEDIA (Medidas para Estimular el Desarrollo de la Industria Audiovisual) conciben el desarrollo del sector audiovisual europeo, no en el binomio I + D, sino a partir del principio político de supeditar la creación cultural a postulados de identidad cultural. Es obvio que en ese conjunto de principios políticos interviene más el 
beneficio mercantil que los postulados científicos. Ante tantas declaraciones fáciles, sería muy interesante desde el punto de vista de la Antropología Cultural y de la Historia (nadie lo va a intentar, por supuesto) desvelar de qué identidades estamos hablando en ocasiones y a quién benefician.

Los criterios de mercado por los que ha apostado la Unión Europea comprometen también a la creación y le sirven de coartada para obtener exenciones y subvenciones. Los guionistas en las II Jornadas de Guión (La televisión de las ideas) (Barcelona, noviembre de 1995) se han mostrado preocupados por la estandardización de los formatos y por los problemas que crean los pools de guionistas. Se quejaron de que el seguimiento mimético de los guionistas de las sitcom americanas e inglesas esté influyendo negativamente en la puesta en marcha de nuevas ideas. ¿Es que no tiene que ver esto con esa cacareada identidad cultural europea? La crisis mundial del cine depende en gran medida de la creciente degradación en la calidad de sus guiones y ésta se ve condicionada por la formación científico-técnica, la capacitación profesional y, en definitiva, la cultura.

Dentro de un mes va finalizar con el siglo el Programa INF02000 de la Comisión de las Comunidades Europeas para fomentar el desarrollo de la industria audiovisual y multimedia europea y su utilización en la mal llamada "sociedad de la información". La política científica y tecnológica marcada por la Unión Europea no ha tenido, ni el coraje, ni la generosidad, ni la habilidad de sumar a sus objetivos la contribución de la Universidad. La determinación de las prioridades de investigación propuestas responde más a criterios profesionales, industriales y de mercado, que a criterios científicos, culturales y sociales. Pesa en exceso la visión de Europa como mercado competitivo frente a la poderosa industria audiovisual norteamericana y pesa también en exceso una subordinación de la creación libre de los productos audiovisuales (y la primera creación realmente libre ha de ser la ciencia) a los escenarios constrictivos y concéntricos de una pluralidad de identidades culturales (la identidad europea, las identidades nacionales, las identidades regionales, etc.

\section{Los centros promotores de la ciencia: Las facultades de Ciencias de la Información}


El rigor, el desarrollo y los beneficios sociales de la investigación científica en Comunicación Audiovisual están directamente relacionados con los centros universitarios que han de promoverla. Desde el momento en que la formación de los periodistas y comunicadores audiovisuales quedó encomendada a la Universidad, hace de esto veinticinco años, se abrió un periodo de ambigüedades y sinrazones, que ha condicionado la investigación y ha costado muy caro a la multitud de alumnos, que, fascinados por ese universo, han optado por seguir sus estudios universitarios.

El primer error que cometieron aquellos interesados reformadores fue suprimir las antiguas escuelas oficiales de formación profesional: la Escuela Oficial de Periodismo, la Escuela Oficial de Cine, la Escuela Oficial de Publicidad, etc. Eso de las Escuelas "Oficiales" tenía cierto regusto fascista, pero, al margen de esa connotación, el hecho significaba que, dado el desinterés manifiesto e incluso suicida del sistema educativo por el universo audiovisual, la Universidad debía asumir la totalidad de la formación de los alumnos a todos los niveles. Se veía obligada a "alfabetizar" sit venia verbo) sobre el universo de la imagen, a impartir conocimientos básicos, medios y universitarios, a formar profesionales y a otorgar la licenciatura y el doctorado. A nadie se oculta que era demasiado pedir.

\section{Postergación y menosprecio del estatuto científico}

Los efectos de aquella decisión han sido demoledores para el desarrollo científico y pedagógico de estos saberes. La Universidad clásica, cuyas carreras responden a conocimientos depurados por una larga tradición científica, no ha acabado de comprender porqué los estudios de Periodismo, Cine, Radio, Televisión o Publicidad llegaban al Alma Mater, cuando en realidad no pasaban de ser oficios dignísimos, pero sin un estatuto epistemológico. No olvidan los prebostes de la vieja universidad que la metafísica occidental condenó a la imagen a los confines del mundo de la futilidad y de la sombra y que los padres creadores de estos oficios eran feriantes, saltimbanquis, trotamundos y "gentes de mal vivir". En el seno de la vetusta universidad clásica siguen apareciendo reticencias, incluso por parte de algunos responsables de la política científica, sobre la oportunidad de dar cobijo a estos estudios con equiparable dignidad al resto de saberes tradicionalmente superiores, como el Derecho, la Filosofía, la Medicina, las Matemáticas o las Ciencias Naturales. 
Algo así como si la investigación aplicada a estos saberes no pasara de ser una metáfora pretenciosa.

\section{Los proyectos científico-pedagógicos}

Privados de esta tradición científica, los profesores que han venido obteniendo sus cátedras en la nueva Facultad de Ciencias de la Información, se han visto forzados a construir sus proyectos científico-pedagógicos sin marcos estables y seguros de referencia epistemológica, lo que ha dado origen a un problema de falta de identidad. O se han entregado a saberes especulativos y teoréticos, espigando en terrenos foráneos y movedizos, poco o nada susceptibles de dar razón de las decisiones prácticas, que necesita el profesional, como la Semiología, o, procedentes del campo profesional del cine, la radio o la televisión, han optado por impartir sus conocimientos del oficio, confundiendo a la Universidad con una Escuela de Formación Profesional.

Los alumnos, que acuden en masa a estudiar Periodismo, Imagen o Publicidad, especialmente sensibilizados por las dificultades de encontrar empleó que dé salida a sus conocimientos, han aceptado de buen grado esa orientación que han dado a sus estudios algunos profesores, pero han echado en cara a la Universidad al mismo tiempo (desde su punto de vista con toda razón) que no les facilite un sistema adecuado de prácticas.

\section{La Universidad y los profesionales de la comunicación audiovisual}

A la pregunta radical "¿Vale algo la ciencia de la comunicación audiovisual?" habría que responder: vale lo que la sociedad y los profesionales estén dispuestos a dar por ella. Los profesionales españoles de la comunicación audiovisual no reconocen el valor de las facultades de Ciencias de la Información. En la mesa redonda sobre La enseñanza del cine convocada en Madrid (diciembre, 1995) un buen número de profesionales, entre ellos varios directores eminentes, relativizaron no sólo el valor formativo de las facultades universitarias, sino también de las escuelas de cine y reivindicaron la figura del meritoriaje al servicio y a las órdenes de un director. Lo mejor de nuestro cine estaba añorando (esto es sintomático) su etapa artesanal. 
La difícil convivencia de la universidad y la profesión del cine ha estado dominada en una y otra parte por un sinfín de complejos. Profesores universitarios de formación teórica y libresca que no son profesionales ni han pisado en su vida un plato; directores de cine, que no han superado el complejo de no haber iniciado o terminado sus estudios universitarios; guionistas que no han podido llegar a directores de cine y críticos que tratan de disimular el complejo de no haber pasado por la escuela o de haber fracasado en ella. Sobre el cimiento de esta variopinta desconfianza y de estos complejos de inferioridad poco positivo se puede construir.

\section{Reivindicación profesional del científico y del docente}

Para la Universidad el concepto de "profesión" no se agota en la práctica socialmente sancionada y reconocida de los oficios de lo audiovisual. Para la Universidad son también profesiones respetables, confiadas a su misión formativa el investigador, el educador y el docente, cuyas tareas se relacionan con el universo audiovisual. ¿Y qué sucede? Sucede que, cuando un conjunto de actitudes soberbias, excluyentes y corporativistas de uno u otro lado, que tienen por único fundamento las respectivas ignorancias, confunde los linderos que marcan las identidades, también se confunden los roles, se invaden los predios del saber y se falta gravemente al respeto. Los profesionales del cine, por ejemplo, se sienten llamados a la docencia, como ha sucedido en la Comunidad de Madrid, inexplicablemente alentados por las autoridades educativas y los alumnos universitarios se lamentan de que la Universidad, una universidad entendida como escuela de formación profesional, no les enseña nada, cuando en realidad quieren decir que aquello que les enseña, no les interesa. Llevan razón. La culpa no es suya, pero tampoco de la Universidad. En el dominio audiovisual la Universidad necesita de manera imperiosa a los profesionales de calidad del cine, la radio, la televisión y la publicidad.

\section{6. "Comunicólogos" y otros improperios}

Para el profesional del cine, la radio o la televisión el "comunicólogo" es una especie que no debe correr peligro de extinción porque pasta en las praderas foráneas de la especulación quimérica y libresca, fomentada en las facultades. Por no ser profesionalmente peligrosa, la especie del "comunicólogo" ibérico merece ser 
oficialmente protegida por la Academia para seguridad y gozo de los que conocen, practican (y ahora también "enseñan") los oficios. El gran valor de los "comunicólogos" desde el punto de vista profesional es que su existencia produce un efecto placebo y redime de cualquier complejo de inferioridad porque demuestran con eficacia la inutilidad de la ciencia. A esta especie ibérica protegida parecen pertenecer los estudiosos de la "teoría y técnica de" (aplicadas a la programación, al guión, a la producción, a la realización, etc.). Son los docentes afiliados a la funesta manía francesa de las "-logias", como diría Seymour Chatman.

\section{7. "No tomarás el sagrado nombre de la ciencia en vano"}

En esa ceremonia de la confusión el altar de la ciencia no ha sido profanado, porque la ciencia conoce sus límites y siempre es indulgente, pero su sagrado nombre ha sido tomado en vano. El saber empírico, alimentado por la desconfianza respecto a toda especulación y toda teoría y por una suficiencia autocomplaciente, ha llevado a los mejores profesionales del universo audiovisual a fundar sus AACCEE (Academia de las Artes y Ciencias Cinematográficas o de la Televisión, al margen de la Facultad y sin contar con ella. ¿Quién ha dicho que en este país no se cotiza la ciencia? ¡Viva Hollywood y sus galas promocionales!.

\section{Los contornos de la silueta científica en comunicación audiovisual}

Con independencia de su reconocimiento científico y social, el verdadero problema de la investigación en comunicación audiovisual es la precariedad de su estatuto epistemológico. La reciente incorporación de estos estudios a la Universidad española (25 años no son nada) han obligado a improvisar un corpus científico que no acaba de perfilar sus linderos respecto a otros saberes de rango superior. Es grave porque la investigación interdisciplinar requiere siempre que los puentes que a la vez vinculan y distancian, unen y diferencian los saberes, sean portadores de significados bien depurados. Sabemos mucho mejor, por ejemplo, lo que la sociología o la antropología aportan al estudio del cine que lo que el cine aporta al estudio de estas disciplinas. El resultado es que no hay una filmología, pero sí hay una historia del cine, una psicología o sociología del cine, etc. El contenido y el método de la mayor parte de las investigaciones científicas sobre comunicación audiovisual han sido focalizados, en efecto, a partir de otras ciencias particulares. 
Por esos derroteros se viene desarrollando ampliamente en Estados Unidos, por ejemplo, desde finales de los años 50 , la investigación sobre los efectos de los medios.

Las investigaciones en comunicación audiovisual permiten distinguir cuatro perfiles y rasgos que configuran su silueta:

a) Muchas de las sedicentes "investigaciones" en comunicación audiovisual, incluidas las tesis doctorales, si tenemos en cuenta sus protocolos y metodologías heurísticas, son más informes o ensayos de divulgación que verdaderas investigaciones científicas.

b) Las investigaciones científicas sobre lo audiovisual erigen en objeto privilegiado y prioritario a la imagen en detrimento del sonido; al cine en detrimento del resto de los medios audiovisuales y a la historia en detrimento de otras disciplinas.

c) Las investigaciones sobre la comunicación audiovisual han dependido con exceso de los modelos del análisis textual: análisis de contenidos, gramatical, estructuralista y semiótico, que en realidad son menos específicos y productivos para entender el universo audiovisual como fenómeno tecnológico, social y cultural.

d) Cuando se han dado intentos metódicos parciales, pero serios, como los de la Escuela de Warburg, para un análisis científico de la imagen, o no se han sabido o no se han querido aplicar al análisis de la obra audiovisual.

\section{El decálogo de la investigación científica}

En este momento de desarrollo tecnológico acelerado, que caracteriza al universo audiovisual, se hace más patente y ostensible el decalage de la investigación científica y las dificultades a la hora de definir sus prioridades con criterios de utilidad social. La aceleración de la industria audiovisual, inserta en macroalianzas con la informática, las telecomunicaciones y el entertainment, no ha permitido a la universidad establecer su thinking time. El uso y consumo de los bienes tecnológicos se atiene a un régimen de individualismo salvaje, limitado únicamente por la priopia iniciativa y la propia intuición. El desarrollo del discurso multimedia (es únicamente 
un ejemplo) parece que debería abordarse dentro del problema de la justificación electiva, de naturaleza científica, en función de una verdadera taxonomía de los medios y soportes empleados. La peor libertad en la combinación de los medios consiste en desnaturalizarlos, sobre todo si se hace por ignorancia, pero a nadie parece preocupar este problema.

En el dominio audiovisual no existe en la práctica investigación básica y son otros, no la propia universidad, los que definen los planes de la investigación científica aplicada, que condiciona el desarrollo tecnológico de los medios, la industria del hardware y del software y, en general, el sentido de su impacto en la sociedad y en la cultura a fines del milenio.

\section{Dos inconvenientes para el desarrollo de la investigación científica en comunicación audiovisual}

El desconocimiento o la insensibilidad de las autoridades académicas, sumados a la atonía y el desconcierto de quienes tendríamos la obligación de haberlo solicitado con vehemencia, han contribuido a consolidar dos graves inconvenientes para el desarrollo de la investigación. El primero ha sido la cohabitación del área de conocimiento y de los departamentos de comunicación audiovisual y de publicidad en facultades de Ciencias de la Información, comúnmente reconocidas como "facultades de periodismo". En este hospedaje indiferenciado y casi parasitario ha echado raíces nuestra falta de identidad y, como consecuencia de ello (segundo inconveniente) nos hemos perdido que una Facultad de Ciencias de la Comunicación, que nos definiría mejor, fuera declarada facultad experimental, lo que hubiera permitido un tratamiento diferente en cuanto a investigación básica y aplicada, en cuanto a un régimen de númerus clausus y en cuanto a la asignación de recursos.

\section{El Área de Conocimiento de Comunicación Audiovisual}

El problema básico y fundamental es sin duda sentar las bases para definir la identidad científica, académica y docente del Área de Conocimiento de Comunicación Audiovisual y Publicidad. Esto, absolutamente necesario, no puede hacerse sino a través del consenso de los departamentos que la integran. La cooperación debería reclamar como paso inmediato una reestructuración de los dos 
departamentos actuales, inspirada únicamente en criterios científicos y académicos, y nunca en criterios políticos o razones de poder. La difícil tarea que se presenta ahora es cómo poder actuar en esa reestructuración si no están disponibles los contenidos que deberían perfilar la oferta diferenciada que los departamento han de hacer a los alumnos, verdaderos protagonistas de la acción formativa.

\section{Los nuevos planes de estudios}

El debate (reforma, contrarreforma o como diablos se la quiera llamar) del plan de estudios deja pocas oportunidades para ese rigor científico y académico que debería presidir las futuras relaciones de los departamentos. La reforma del plan de estudios acometida por la Universidad española es una torpeza increíble, que solo puede justificar la demagogia política. La política científica y educativa impone como criterio básico que la elaboración de un nuevo plan de estudios universitarios (asunto grave $y$, por consiguiente, excepcional) debe ser la última de las medidas de una reforma. Es la guinda que colma la tarta de una renovación: la propuesta concreta que cristaliza, consolida y da garantías de eficacia a una reforma general, que llega, al fin, a sus verdaderos actores y destinatarios: los alumnos y, a través de ellos, a la sociedad. La torpeza de los reformadores ha consistido en invertir exactamente los términos y pensar que reformar los planes de estudio, sin reformar previamente las estructuras, las condiciones y métodos de trabajo, la asignación de los recursos requeridos, la preparación investigadora y docente, la selección y la retribución económica y sicológica del profesorado, la reducción de la ratio profesor-alumnos, moral del profesorado, la creación de nuevas condiciones de trabajo, etc.... podría servir para algo. Para lo único que sirve en realidad es para un lema político y para encubrir el paro juvenil.

La sedicente reforma del plan de estudios ha servido en realidad para poner de manifiesto la escasa sensibilidad social, la esclerosis burocrática, las actitudes inmovilistas y las ambiciones de poder de unos pocos, más dispuestos a perpetuar posiciones de privilegio que a responder a las necesidades laborales, económicas y culturales de nuestra sociedad en cambio acelerado. Es lo único que han hecho y probablemente lo único que podían hacer, porque (¡que quede claro!): los profesores no son los responsables del fracaso del plan de estudios, del anterior y del que viene. Son, si lo miramos bien, junto con los alumnos, sus verdaderas víctimas. 


\section{La enseñanza de la comunicación audiovisual en otros niveles del sistema educativo}

En el pliego de cargos con que denunciamos la penosa situación de la investigación en comunicación audiovisual hay que incluir la necesidad de que la investigación científica sirva de base para la innovación pedagógica. La enseñanza del cine, la radio, la televisión ( $\mathrm{y}$, en general, de los medios audiovisuales y de la publicidad) responde hoy a imperiosas necesidades de formación básica de todos los españoles, que solo será posible, si el sistema educativo, cerrado y paralizado por la esclerosis burocrática y la demagogia política, comienza a comportarse como un sistema abierto a la comunicación, a la cultura de actualidad y al mercado laboral.

La prioridad de la formación literaria carece de sentido en un mundo que hoy está dominado por los mensajes audiovisuales, que, como vio con acierto Georges Friedman en los años 60, constituyen una verdadera "escuela paralela" para el ciudadano contemporáneo. El hecho de que el ciudadano carezca de formación crítica frente a los poderosos medios de comunicación constituye un atentado a su libertad individual y al conjunto de los valores que constituyen la cultura, que el viejo sistema educativo ha transmitido durante siglos. La insensibilidad de la dictadura franquista ante este rearme espiritual del ciudadano tenía cierta lógica. No la tiene, en cambio, el hecho sorprendente de que aquella insensibilidad se ha perpetuado en una democracia, que, al parecer, se siente cómoda asentándose en votantes anestesiados por la propaganda y poco pertrechados de defensas críticas para reaccionar a los excesos del poder mediático y a los mensajes interesados del cine, la radio, la televisión o la publicidad.

La enseñanza de la comunicación audiovisual en los niveles anterior al universitario aportaría tres ventajas:

a) En primer lugar, ayudaría a la Universidad a redefinir su propuesta docente en el nivel superior que le es propio, ya que los alumnos llegarían con una preparación previa, que le permitiría profundizar en los estudios y dedicarse más y mejor a la investigación. 
b) En segundo lugar, la Universidad se dedicaría a formar profesorado en comunicación audiovisual y, por consiguiente, abriría una nueva salida a quienes cursan estos estudios;

c) En tercer lugar, la Universidad daría a sus conocimientos una utilidad social de gran trascendencia para la vida de los ciudadanos en una democracia avanzada.

Son inculpaciones, proyectos y deseos, todo un pliego de cargos, para provocar una reacción de nuestras conciencias, adormecidas por la rutina o por el interés mercantil en pro del desarrollo de un tipo de saber científico, en el que, a pesar de todo, creemos, por el que hemos apostado, pero lleno de asechanzas y tribulaciones. "Ojalá el cielo -terminaré con la misma sentencia de Nieburg- nos conceda la serenidad para aceptar las cosas que no se pueden cambiar; el coraje para cambiar todo lo que se puede y se debe; y la inteligencia para discernir entre ambas cosas".

\section{BIBLIOGRAFÍA}

GARCÍA JIMÉNEZ, J. (1993). Narrativa audiovisual. Madrid: Cátedra.

CHATMAN, S. (1990). Historia y discurso. Madrid: Taurus.

LAUREL, B. (1990). The Art of human-computer interface design. Readign: AddisonWesley Publishing Company.

PUPPO, F. (1997) "La Pantalla: espejo del alma”, en: ESCUDERO, L.; VERÓN, E., comps. Telenovela: ficción popular y mutaciones culturales. Barcelona: Ed. Gedisa. p. $113-120$

MORLEY, D. (1992) Television, audiences \& cultural studies . London: Routledge. [trad. Esp..: MORLEY, D. Televisión, audiencias y estudios culturales. Buenos Aires: Amorrortu, 1996]. 